\title{
SPATIAL DECAY OF TRANSIENT END EFFECTS IN FUNCTIONALLY GRADED HEAT CONDUCTING MATERIALS
}

\author{
BY \\ C. O. HORGAN (Applied Mechanics Program, Department of Civil Engineering, University of \\ Virginia, Charlottesville, VA 22903, USA) \\ AND \\ R. QUINTANilla (Matematica Aplicada 2, E.T.S.E.I.T.- U.P.C., Colom 11, 08222 Terrassa, \\ Barcelona, Spain)
}

\begin{abstract}
The purpose of this research is to investigate the influence of material inhomogeneity on the spatial decay of end effects in transient heat conduction for isotropic inhomogeneous heat conducting solids. The work is motivated by the recent research activity on functionally graded materials (FGMs), i.e., materials with spatially varying properties tailored to satisfy particular engineering applications. The spatial decay of solutions to an initial-boundary value problem with variable coefficients on a semi-infinite strip is investigated. It is shown that the spatial decay of end effects in the transient problem is faster that that for the steady-state case. Qualitative methods involving second-order partial differential inequalities for quadratic functionals are first employed. Explicit decay estimates are then obtained by using comparison principle arguments involving solutions of the one-dimensional heat equation with constant coefficients. The results may be interpreted in terms of a Saint-Venant principle for transient heat conduction in inhomogeneous solids.
\end{abstract}

1. Introduction. Functionally graded composite materials (FGMs) have attracted considerable attention in recent years. These materials are characterized by a microstructure that is spatially variable on a macroscale and were initially developed for high temperature applications. Recent surveys are given by Erdogan [7], Jin and Batra [20] and Aboudi et al. [23]. A commonly used FGM, for example, is a metal/ceramic composite designed to provide superior oxidation and thermal shock resistance. The material is continuously graded to transition from a high strength, high toughness metallic core to an efficient thermally shielding ceramic surface. FGMs are being used as interfacial zones to improve the bonding strength of layered composites, to reduce the residual and

Received February 7, 2000.

2000 Mathematics Subject Classification. Primary 74E05, 74G50, 80A20, 35K05.

(C)2001 Brown University 
thermal stresses in bonded dissimilar materials and as wear resistant layers in machine and engine components.

The mechanical and mathematical modeling of FGMs is currently an active research area. When a continuum mechanics approach is appropriate, the models involve nonhomogeneous materials with continuously varying properties. Fracture mechanics of FGMs using this viewpoint is discussed, for example, by Erdogan [7] and by Jin and Batra [20]. Some other fundamental problems that have been studied recently using nonhomogeneous elasticity theory are those of thick plate theory (Abid Mian and Spencer [1]), torsion (Rooney and Ferrari [25], Horgan and Chan [11]), elastic vibrations (Loy et al. [22], Horgan and Chan [12]) and the analysis of Saint-Venant end effects (Horgan and Payne [15], Horgan and Miller [14], Scalpato and Horgan [26], Chan and Horgan [6], Horgan and Quintanilla [17]). Since the mathematical problems arising are complicated, much of the work on FGMs has been carried out numerically, e.g., using finite elements (FEM). It is necessary to also develop analytical approaches for such problems. Analytical solutions to benchmark problems provide invaluable checks on the accuracy of numerical or approximate analytical schemes and allow for widely applicable parametric studies.

The purpose of the present paper is to investigate analytically another fundamental issue for FGMs, namely the extent of end effects in transient heat conduction problems. For homogeneous heat conducting solids, this question was first raised by Boley (see, e.g., Boley [3], Boley and Weiner [4]) in the context of a Saint-Venant principle for heat conduction. Saint-Venant's principle in the equilibrium theory of linear elasticity has a long history (see, e.g., the reviews by Horgan and Knowles [13], Horgan [9,10]) and is a consequence of the elliptic character of the governing partial differential equations. The study of related issues for parabolic equations is more recent. Using explicit upper bounds for solutions of half-space problems obtained from appropriate fundamental solutions, Boley [3] observed that the spatial influence of transient effects was even more localized than that of the steady-state. The validity of a Saint-Venant principle of the more traditional type, for two-dimensional rectangular or three-dimensional cylindrical domains subject to nonzero boundary conditions on the ends only, was considered by Boley and Weiner [4]. An illustrative example involving the explicit solution of a specific initial-boundary value problem for a semi-infinite rectangular strip is discussed by Boley and Weiner [4], pp. 186-187. In this example, it is seen that the spatial decay of end effects at any time $t$ in the transient problem is faster than that for the steady-state case. Considerable work has been done on examining the decay of end effects for initial-boundary value problems for parabolic equations on cylindrical-type domains subject to nonzero boundary conditions only on the ends. Qualitative methods, analogous to those developed for elliptic equations, are used to establish the exponential decay of solutions of such problems with distance from the ends and estimates for the decay rates are obtained. For classical linear heat conduction in homogeneous solids, Knowles [21] established that end effects for the transient case decay spatially at least as rapidly as do their counterparts in the steady-state case. This was established by Knowles [21] using arguments based on differential inequalities for quadratic functionals. A similar result was obtained by Horgan and Wheeler [18] using the maximum principle. A stronger result, analogous to that of 
Boley [3], Boley and Weiner [4], was established by Horgan et al. [16], who showed that the spatial decay of end effects at each fixed time $t$ in the transient problem is faster than that for the steady-state case. This work was extended to classes of nonlinear heat equations by Quintanilla [24], where references to related research may be found. Our purpose here is to establish a similar result for functionally graded materials. We note that a spatial decay estimate for a diffusion equation with variable coefficients was obtained by Franchi and Straughan [8], whose main emphasis, however, was on results for improperly posed, backward-in-time problems. A recent paper by Ignaczak [19] was concerned with decay estimates for one-dimensional heat conduction in inhomogeneous half-spaces.

In the next section, we formulate the basic problem to be examined. We consider an initial-boundary value problem for a (generalized) heat equation with variable coefficients on a semi-infinite two-dimensional strip. The long sides of the strip are maintained at zero temperature, while a prescribed temperature is given at the near end. In Sec. 3, the special case of a laterally inhomogeneous material is considered. A second-order partial differential inequality for a quadratic measure of the temperature is established. In Sec. 4, the same differential inequality is established for the general inhomogeneous material, using a change dependent variable. Such a change of variable has been recently used to analyze end effects for the elliptic case (Chan and Horgan [6]). In Sec. 5, on assuming that the temperature satisfies an appropriate asymptotic behavior as the axial variable tends to infinity, it is shown that solutions to the differential inequality may be estimated in terms of solutions to the one-dimensional heat equation with constant coefficients. The main results of the paper are obtained in Sec. 6 providing an explicit estimate for the solution which shows that the spatial decay of end effects is faster than that of the steady-state. The influence of material inhomogeneity on the rate of decay of end effects is discussed in Sec. 7. For simplicity of exposition, we confine attention to the two-dimensional problem on a semi-infinite strip. The results may be easily extended to three-dimensional problems on cylindrical domains.

2. Formulation of the problem. We are concerned with solutions of the heat equation for an isotropic inhomogeneous heat conducting material in two dimensions. If $u(\mathbf{x}, t)=u\left(x_{1}, x_{2}, t\right)$ is the temperature field, then $u$ satisfies the (generalized) heat equation

$$
\frac{\partial}{\partial x_{1}}\left(K(\mathbf{x}) \frac{\partial u}{\partial x_{1}}\right)+\frac{\partial}{\partial x_{2}}\left(K(\mathbf{x}) \frac{\partial u}{\partial x_{2}}\right)=c(\mathbf{x}) \rho(\mathbf{x}) \frac{\partial u}{\partial t}
$$

on $R \times(0, \infty)$ where $R$ is the semi-infinite strip $R=\left\{\left(x_{1}, x_{2}\right) \mid x_{1}>0,0<x_{2}<h\right\}$. In (2.1), $K(\mathbf{x})$ is the thermal conductivity, $c(\mathbf{x})$ is the specific heat, $\rho(\mathbf{x})$ is the density, and these quantities are assumed to be positive, uniformly bounded functions on $R$. It is also convenient for later purposes to introduce the heat diffusivity coefficient defined by

$$
\kappa(\mathbf{x})=\frac{K(\mathbf{x})}{\rho(\mathbf{x}) c(\mathbf{x})} .
$$

We assume that

$$
0<K_{m} \leq K(\mathbf{x}) \leq K_{M}, \quad 0<\kappa_{m} \leq \kappa(\mathbf{x}) \leq \kappa_{M}, \mathbf{x} \in R .
$$


The lateral sides of the strip are maintained at zero temperature, the temperature is zero initially, and the end $x_{1}=0$ is subject to a prescribed temperature so that

$$
\begin{gathered}
u\left(x_{1}, 0, t\right)=0, \quad u\left(x_{1}, h, t\right)=0, \quad x_{1} \geq 0, \quad t \geq 0, \\
u(\mathbf{x}, 0)=0, \quad \mathbf{x} \in R, \\
u\left(0, x_{2}, t\right)=f\left(x_{2}, t\right), \quad 0 \leq x_{2} \leq h, \quad t \geq 0,
\end{gathered}
$$

where $f\left(x_{2}, t\right)$ is sufficiently smooth and is such that

$$
f\left(x_{2}, 0\right)=0, \quad 0 \leq x_{2} \leq h .
$$

It is assumed that $f$ is such that a classical solution $u$ of the initial-boundary value problem (2.1)-(2.6) exists.

For a homogeneous heat conducting material where $K, c$ and $\rho$ are constants, it has been shown by Knowles [21] that, if $u \rightarrow 0$ (uniformly in $x_{2}, t$ ) as $x_{1} \rightarrow \infty$, then at each instant, $u(\mathbf{x}, t)$ decays spatially in $x_{1}$ at least at an exponential rate identical to that of the steady-state problem. A sharper result was obtained by Horgan et al. [16] who showed that solutions decay at a rate that is faster than the exponential decay predicted by Knowles [21]. Our purpose here is to establish an analog of the result of Horgan et al. [16] for the inhomogeneous material and to assess the effects of material inhomogeneity on the rate of decay of end effects.

3. Laterally inhomogeneous materials. It is instructive to first consider the special case of laterally inhomogeneous materials where

$$
K(\mathbf{x})=K\left(x_{2}\right), \quad c(\mathbf{x}) \rho(\mathbf{x})=c\left(x_{2}\right) \rho\left(x_{2}\right) .
$$

For $u(\mathbf{x}, t)$ satisfying $(2.1),(2.4)-(2.6)$, we define the (nonnegative) functional

$$
P(z, t)=[J(z, t)]^{1 / 2} \equiv\left(\int_{0}^{t} \int_{L_{z}} K u^{2} d x_{2} d \tau\right)^{1 / 2},
$$

where $L_{z}$ denotes the line segment $\left\{x_{1}=z, 0 \leq x_{2} \leq h\right\}$. In this section, we establish that $P(z, t)$ satisfies the second-order differential inequality

$$
P_{z z} \geq k P+d \dot{P}, \quad z>0, t>0,
$$

where the subscript denotes differentiation with respect to the spatial variable $z$ and the dot denotes differentiation with respect to time. In (3.3), $k$ and $d$ are positive constants which will be defined below.

The derivation of (3.3) proceeds as follows: From its definition in (3.2), and the first of (3.1), it follows that

$$
\begin{gathered}
J_{z}=\int_{0}^{t} \int_{L_{z}}\left(2 u K u_{, 1}\right) d x_{2} d \tau \\
J_{z z}=2 \int_{0}^{t} \int_{L_{z}}\left[u\left(K u_{, 1}\right)_{, 1}+K u_{, 1}^{2}\right] d x_{2} d \tau
\end{gathered}
$$


where the comma denotes differentiation with respect to the indicated coordinate. On using the differential equation (2.1), the divergence theorem and the boundary conditions (2.4), we get

$$
J_{z z}=2 \int_{0}^{t} \int_{L_{z}}\left(K u_{, 2}^{2}+K u_{, 1}^{2}+c \rho u \dot{u}\right) d x_{2} d \tau .
$$

Since $u$ is zero at the end points of $L_{z}$ by virtue of (2.4), we have, at each fixed $t$, the inequality

$$
\int_{L_{z}} K\left(x_{2}\right) u_{, 2}^{2} d x_{2} \geq \lambda_{1} \int_{L_{z}} K\left(x_{2}\right) u^{2} d x_{2}
$$

for $z \geq 0$, where $\lambda_{1}$ is the smallest positive eigenvalue of

$$
\begin{gathered}
\frac{d}{d x_{2}}\left(K\left(x_{2}\right) \frac{d \phi}{d x_{2}}\right)+\lambda K\left(x_{2}\right) \phi=0, \\
\phi(0)=0, \phi(h)=0 .
\end{gathered}
$$

Thus, on using (3.7) in (3.6), it follows that

$$
J_{z z} \geq 2 \lambda_{1} J+2 \int_{0}^{t} \int_{L_{z}} K u_{, 1}^{2} d x_{2} d \tau+2 \int_{0}^{t} \int_{L_{z}} c \rho u \dot{u} d x_{2} d \tau .
$$

Now

$2 \int_{0}^{t} \int_{L_{z}} c \rho u \dot{u} d x_{2} d \tau=\int_{0}^{t} \frac{\partial}{\partial \tau}\left(\int_{L_{z}} c \rho u^{2} d x_{2}\right) d \tau=\int_{L_{z}} c \rho u^{2}(\mathbf{x}, t) d x_{2}-\int_{L_{z}} c \rho u^{2}(\mathbf{x}, 0) d x_{2}$

$$
=\int_{L_{z}} c \rho u^{2}(\mathbf{x}, t) d x_{2}
$$

where the initial condition (2.5) has been used to obtain the last step in (3.11). On employing (3.11) in (3.10), we get

$$
J_{z z} \geq 2 \lambda_{1} J+2 \int_{0}^{t} \int_{L_{z}} K u_{, 1}^{2} d x_{2} d \tau+\int_{L_{z}} c \rho u^{2} d x_{2} .
$$

Since $J \geq 0$, we deduce from (3.4) and (3.12) that

$$
\begin{gathered}
J J_{z z}-\left(J_{z}\right)^{2} / 2 \geq 2\left[\left(\int_{0}^{t} \int_{L_{z}} K u^{2} d x_{2} d \tau\right)\left(\int_{0}^{t} \int_{L_{z}} K u_{, 1}^{2} d x_{2} d \tau\right)\right. \\
\left.-\left(\int_{0}^{t} \int_{L_{z}} K u_{, 1} u d x_{2} d \tau\right)^{2}\right]+2 \lambda_{1} J^{2}+J \int_{L_{z}} c \rho u^{2} d x_{2} \\
\geq 2 \lambda_{1} J^{2}+J \int_{L_{z}} \frac{K}{\kappa} u^{2} d x_{2},
\end{gathered}
$$

where Schwarz's inequality has been used to obtain (3.14) and we have also used (2.2) to rewrite the last integral. The final step in the derivation of (3.3) requires an estimate for the last integral on the right in (3.14). By virtue of its definition in (3.2), we have

$$
\dot{J}=\int_{L_{z}} K u^{2} d x_{2}
$$


On using the right-hand side of $(2.3)_{2}$ in (3.14) we then obtain

$$
J J_{z z}-\left(J_{z}\right)^{2} / 2 \geq 2 \lambda_{1} J^{2}+\kappa_{\Lambda I}^{-1} J \dot{J} .
$$

On recalling the definition of $P(z, t)$ in (3.2), it may be verified that (3.16) and (3.3) are equivalent where the positive constants $k$ and $d$ are given by

$$
k=\lambda_{1},
$$

and

$$
d=\kappa_{M}^{-1}
$$

respectively. This completes the derivation of (3.3).

Observe that no assumption has yet been made on the behavior of $u$ as $x_{1} \rightarrow \infty$.

4. General inhomogeneity; a change of variable. In this section, we derive a differential inequality of the form (3.3) for generally inhomogeneous materials where

$$
K(\mathbf{x})=K\left(x_{1}, x_{2}\right), \quad c(\mathbf{x}) \rho(\mathbf{x})=c\left(x_{1}, x_{2}\right) \rho\left(x_{1}, x_{2}\right) .
$$

For solutions $u(\mathbf{x}, t)$ of $(2.1),(2.4)-(2.6)$, the functional $P(z, t)$ is again defined as in (3.2). To establish the desired result (3.3), we first introduce a new dependent function $W(\mathbf{x}, t)$ by setting

$$
u(\mathbf{x}, t)=W(\mathbf{x}, t) K^{-1 / 2}(\mathbf{x}) .
$$

Then one can readily verify that $(2.1)$ is equivalent to

$$
\nabla^{2} W+\frac{1}{4 K^{2}}\left[\left(K_{, 1}^{2}+K_{, 2}^{2}\right)-2 K\left(K_{, 11}+K_{, 22}\right)\right] W=\kappa^{-1} \frac{\partial W}{\partial t}, \text { on } R .
$$

The boundary conditions $(2.4),(2.6)$ and the initial condition (2.5) become

$$
\begin{gathered}
W\left(x_{1}, 0, t\right)=W\left(x_{1}, h, t\right)=0, \quad x_{1} \geq 0, t \geq 0, \\
W\left(0, x_{2}, t\right)=K^{1 / 2}\left(0, x_{2}\right) f\left(x_{2}, t\right), \quad 0 \leq x_{2} \leq h, \quad t \geq 0,
\end{gathered}
$$

and

$$
W(\mathbf{x}, 0)=0, \mathbf{x} \in R,
$$

respectively. It is convenient to write the partial differential equation (4.3) for $W$ as

$$
\nabla^{2} W+F\left(K\left(x_{1}, x_{2}\right)\right) W=\kappa^{-1} \dot{W} \text { on } R
$$

where

$$
\begin{aligned}
F(K) & =\frac{1}{4 K^{2}}\left[|\nabla K|^{2}-2 K \nabla^{2} K\right] \\
& \equiv-K^{-1 / 2} \nabla^{2}\left(K^{1 / 2}\right) .
\end{aligned}
$$

The asymptotic behavior of solutions $u$ to the problem (2.1), (2.4)-(2.6) may now be deduced from the asymptotic behavior of solutions $W$ to (4.7), (4.4)-(4.6).

By virtue of (4.2) and (3.2) we have

$$
P(z, t)=[J(z, t)]^{1 / 2}=\left(\int_{0}^{t} \int_{L_{z}} W^{2} d x_{2} d \tau\right)^{1 / 2}
$$


so that

$$
\begin{gathered}
J_{z}=\int_{0}^{t} \int_{L_{z}} 2 W W_{, 1} d x_{2} d \tau \\
J_{z z}=2 \int_{0}^{t} \int_{L_{z}}\left(W W_{, 11}+W_{, 1}^{2}\right) d x_{2} d \tau .
\end{gathered}
$$

On using the differential equation (4.7), the divergence theorem and the boundary conditions (4.4), we obtain

$$
J_{z z}=2 \int_{0}^{t} \int_{L_{z}}\left[W_{, 2}^{2}+W_{, 1}^{2}-F W^{2}+\kappa^{-1} W \dot{W}\right] d x_{2} d \tau .
$$

Since $W$ is zero at the end points of $L_{z}$ by virtue of (4.4), we have, at each fixed $t$, the Wirtinger inequality

$$
\int_{L_{z}} W_{, 2}^{2} d x_{2} \geq \lambda_{1}^{*} \int_{L_{z}} W^{2} d x_{2}
$$

for $z \geq 0$, where

$$
\lambda_{1}^{*}=\frac{\pi^{2}}{h^{2}}
$$

On using (4.14) in (4.13) we obtain

$$
J_{z z} \geq 2 \int_{0}^{t} \int_{L_{z}}\left(\lambda_{1}^{*}-F\right) W^{2} d x_{2} d \tau+2 \int_{0}^{t} \int_{L_{z}} W_{, 1}^{2} d x_{2} d \tau+2 \int_{0}^{t} \int_{L_{z}} \kappa^{-1} W \dot{W} d x_{2} d \tau
$$

The last term in (4.16) is treated just as in (3.11). Thus, on using the initial condition (4.6), it is easily verified that

$$
2 \int_{0}^{t} \int_{L_{z}} \kappa^{-1} W \dot{W} d x_{2} d \tau=\int_{L_{z}} \kappa^{-1} W^{2} d x_{2}
$$

The first term in (4.16) is such that

$$
2 \int_{0}^{t} \int_{L_{z}}\left(\lambda_{1}^{*}-F\right) W^{2} d x_{2} d \tau \geq 2 k \int_{0}^{t} \int_{L_{z}} W^{2} d x_{2} d \tau
$$

where the constant $k$ is given by

$$
k=\inf _{R}\left(\frac{\pi^{2}}{h^{2}}-F\right)
$$

and we recall the definition of $F(K) \equiv F\left(x_{1}, x_{2}\right)$ in (4.8), (4.9). Since the remainder of the argument requires that $k$ be positive, it is assumed henceforth that

$$
\sup _{R} F<\frac{\pi^{2}}{h^{2}} \text {. }
$$

On employing (4.17) and (4.18) in (4.16) we obtain

$$
J_{z z} \geq 2 k J+2 \int_{0}^{t} \int_{L_{z}} W_{, 1}^{2} d x_{2} d \tau+\int_{L_{z}} \kappa^{-1} W^{2} d x_{2}
$$


Since $J \geq 0$, it follows from (4.11) and (4.21) and Schwarz's inequality that

$$
J J_{z z}-\left(J_{z}\right)^{2} / 2 \geq 2 k J^{2}+J \int_{L_{z}} \kappa^{-1} W^{2} d x_{2} .
$$

Since

$$
\dot{J}=\int_{L_{z}} W^{2} d x_{2}
$$

we use $(2.2),(2.3)_{2}$ to deduce from $(4.22)$ that

$$
J J_{z z}-\left(J_{z}\right)^{2} / 2 \geq 2 k J^{2}+\kappa_{\Lambda I}^{-1} J \dot{J}
$$

which has the same form as (3.16). Thus, as in Sec. 3, we find that

$$
P_{z z} \geq k P+d \dot{P}, \quad z>0, \quad t>0,
$$

where $k>0$ is defined in (4.19) and

$$
d=\kappa_{M I}^{-1}
$$

The inequality (4.25) has exactly the same form as (3.3) with the same constant $d$ and with $k$ given by (4.19). This completes the proof of (4.25), valid for the generally inhomogeneous materials (4.1) and established under the additional assumption (4.20). Again we note that no assumption on the behavior of $u$ as $x_{1} \rightarrow \infty$ has been necessary in the derivation of $(4.25)$.

5. A comparison result for solutions of (3.3), (4.25). In this section, we show that the function $P(z, t)$ satisfying (3.3) or equivalently (4.25) can be bounded above by the solution to a related initial-boundary value problem for the one-dimensional heat equation. The argument here follows that of Horgan et al [16]. By virtue of its definition, $P(z, t)$ satisfies the initial condition

$$
P(z, 0)=0, \quad z \geq 0
$$

and the boundary condition

$$
P(0, t)=\left(\int_{0}^{t} \int_{L_{0}} K\left(0, x_{2}\right) f^{2} d x_{2} d \tau\right)^{1 / 2} \equiv g(t) \geq 0, \quad t \geq 0
$$

where

$$
g(0)=0 .
$$

We now assume the following asymptotic behavior for $P(z, t)$ :

$$
P(z, t) \rightarrow 0 \quad \text { (uniformly in } t \text { ) as } z \rightarrow \infty .
$$

Thus the temperature field satisfying (2.2), (2.4)-(2.6) is assumed to vanish in a weighted mean-square sense as the axial variable tends to infinity.

Let

$$
P(z, t)=\exp \left(-\frac{k t}{d}\right) v(z, t)
$$

Then, it follows from (3.3) (or (4.25)), (5.1), (5.2) and (5.4) that $v(z, t) \geq 0$ and satisfies

$$
\mathcal{L} v \equiv v_{z z}-d \dot{v} \geq 0, \quad z>0, \quad t>0,
$$




$$
\begin{gathered}
v(z, 0)=0, \quad z \geq 0, \\
v(0, t)=\exp \left(\frac{k t}{d}\right) g(t) \geq 0, \quad t \geq 0, \\
v(z, t) \rightarrow 0 \quad \text { (uniformly in } t) \quad \text { as } z \rightarrow \infty .
\end{gathered}
$$

An upper bound for $v(z, t)$ in terms of the solution of an initial-boundary value problem for the one-dimensional heat equation now follows immediately from the maximum principle. Let $w(z, t)$ be such that

$$
\begin{gathered}
\mathcal{L} w=0, \quad z>0, \quad t>0, \\
w(z, 0)=v(z, 0)=0, \quad z \geq 0, \\
w(0, t)=v(0, t)=\exp \left(\frac{k t}{d}\right) g(t), \quad t \geq 0, \\
w(z, t) \rightarrow 0 \text { (uniformly in } t \text { ) as } z \rightarrow \infty .
\end{gathered}
$$

The maximum principle for the heat equation now yields

$$
v \leq w, \quad z \geq 0, \quad t \geq 0
$$

and so, from (5.5), we find that

$$
P(z, t) \leq \exp \left(-\frac{k t}{d}\right) w(z, t) .
$$

In the next section, we provide an explicit representation for the unique solution $w(z, t)$ of the problem (5.10)-(5.13) and thus obtain, from (5.15), an explicit upper bound for $P(z, t)$.

6. Spatial decay estimates. The solution to the initial-boundary value problem (5.11)-(5.14) for the one-dimensional heat equation is, of course, well known and can be found in standard textbooks. The representation for $w(z, t)$ that is useful for our purposes (see Carslaw and Jaeger [5], p. 64) is

$$
w(z, t)=\exp \left(\frac{k t}{d}\right) g(t) G(z, t) .
$$

where the nonnegative function $G(z, t)$ is given by

$$
\begin{aligned}
2 G(z, t)= & \exp (-\sqrt{k} z) \operatorname{erfc}\left\{\frac{d^{1 / 2} z}{2 t^{1 / 2}}-\left(\frac{k t}{d}\right)^{1 / 2}\right\} \\
& +\exp (\sqrt{k} z) \operatorname{erfc}\left\{\frac{d^{1 / 2} z}{2 t^{1 / 2}}+\left(\frac{k t}{d}\right)^{1 / 2}\right\} .
\end{aligned}
$$

Here the complementary error function $\operatorname{erfc}(x)$ is defined by

$$
\operatorname{erfc}(x)=2(\pi)^{-1 / 2} \int_{x}^{\infty} e^{-s^{2}} d s
$$


Thus, on using (5.15), we get the upper bound

$$
P(z . t) \leq g(t) G(z . t) .
$$

On recalling the definition of $P(z, t)$ in (3.2) and the notation introduced in (5.2), the result (6.4) can be written directly in terms of the solution $u(\mathrm{x}, t)$ to the original problem (2.1)-(2.6) as

$$
\left(\int_{0}^{t} \int_{L_{z}} K u^{2} d x_{2} d \tau\right)^{1 / 2} \leq\left(\int_{0}^{t} \int_{L_{0}} K\left(0, x_{2}\right) f^{2} d x_{2} d \tau\right)^{1 / 2} G(z, t), \quad z \geq 0, \quad t \geq 0
$$

where $G(z, t)$ is given in $(6.2)$.

The result (6.5) provides a weighted mean-square estimate for the solution $u$ of (2.1)(2.6), subject to the hypothesis (5.4). For the generally inhomogeneous material considered in Sec. 4, the additional assimption (4.20) is also made. Results such as (6.5) may be used to obtain pointuise estimates, uniformly valid on $R \times[0, \infty)$ (sec, e.g., Horgan and Knowles [13]), but this is not our main concern in this paper. Rather we wish to assess the spatial decay character of the estimate (6.5) and contrast it with the corresponding known result for homogeneous materials.

The arguments in Horgan et al. [16] can be used to show that the estimate (6.5) implies that the spatial decay of end effects in the transient problem is faster than that for the steady-state. To sec this, we use the fact that

$$
\lim _{x \rightarrow-\infty} \operatorname{erfc}(x)=2, \quad \lim _{x \rightarrow \infty} \operatorname{erfc}(x)=0,
$$

and so, since $G(z, t)$ is monotonically increasing in $t$, we obtain

$$
G(z, t) \leq \lim _{\tau \rightarrow \infty} G(z, \tau)=\exp (-\sqrt{k} z)
$$

Thus, on using (6.7) in (6.5), we have established that the rate of spatial decay is at least as fast as

$$
\exp (-\sqrt{k} z)
$$

where $k$ is given by (3.17) or (4.19). This decay rate is shown by Chan and Horgan [6] to be the (optimal) decay rate for the steady-state problem, i.e., (2.1)-(2.4), (2.6) for $u(\mathbf{x})$. The foregoing result is the counterpart, for inhomogeneous bodies, of a result due to Knowles [21] for the homogeneous case.

The sharper estimate mentioned at the beginning of the preceding paragraph follows on using a sharper bound than (6.7). We employ the inequality

$$
\sqrt{\pi} \operatorname{erfc}(x)<\frac{1}{x} \exp \left(-x^{2}\right), \quad x>0,
$$

(see Abramowitz and Stegun [2], p. 298) in (6.2). Thus, for $z>2 \sqrt{k} t / d,(6.2)$ and $(6.5)$ yield the estimate

$$
\begin{aligned}
& \left(\int_{0}^{t} \int_{L_{z}} K u^{2} d x_{2} d \tau\right)^{1 / 2} \leq\left(\int_{0}^{t} \int_{L_{z}} K\left(0 . x_{2}\right) f^{2} d x_{2} d \tau\right)^{1 / 2} \\
& \quad \times\left[\frac{2 d^{3 / 2} z(t / \pi)^{1 / 2} \exp (-k \cdot t / d)}{d^{2} z^{2}-4 k t^{2}} \exp \left(-\frac{d z^{2}}{4 t}\right)\right] .
\end{aligned}
$$


The result (6.10) shows that, for fixed the spatial decay is ultimately controlled by the factor

$$
\exp \left(-\frac{d z^{2}}{4 t}\right)
$$

rather than the factor $\exp (-\sqrt{k} z)$ found in the steady state case. The constant $d$ in (6.11) is given by (3.18) or (4.26), that is,

$$
d=\kappa_{M}^{-1},
$$

where we recall that $\kappa(\mathbf{x})$ is the heat diffusivity coefficient defined in (2.2).

7. Discussion. We consider first the special case of a homogeneous isotropic material so that $c, \rho$ and $K$ and thus the diffusivity $\kappa$ are constants and so $d=\kappa^{-1}$. The estimate (6.10) with decay controlled by

$$
\exp \left(-\frac{z^{2}}{4 \kappa t}\right)
$$

then reduces to the two-dimensional version of the result of Horgan et al. [16] established for three-dimensional cylindrical bodies (cf. Ignaczak [19] for half-space problems). Similarly, the weaker estimate with decay as in (6.8) reduces to the two-dimensional version of Knowles' [21] three-dimensional result. For the homogeneous material, the decay rate $\sqrt{k}$ appropriate for the steady-state is given by (3.17) where $\lambda_{1}=\pi^{2} / h^{2}$ (or by (4.19) with $F=0$ ) so that

$$
\sqrt{k}=\frac{\pi}{h}
$$

The decay rate (7.2) is, in fact, optimal for the steady-state problem (see, e.g., Horgan and Knowles [13], Horgan [9, 10]).

Returning to the inhomogeneous material, we first consider the result established after (6.7) that the rate of spatial decay for the transient problem is at least as fast as that for the steady state with decay rate as in (6.8). An extensive study of the dependence of $\sqrt{k}$ on the material inhomogeneity was carried out by Scalpato and Horgan [26], Chan and Horgan [6], and Horgan and Quintanilla [17] in the context of Saint-Venant's principle in anti-plane shear of inhomogeneous isotropic linearly elastic materials. For the steadystate problem, the only variable coefficient that arises is the thermal conductivity $K(\mathbf{x})$. Several illustrative examples for $K(\mathbf{x})$ with power-law or exponential dependence on the coordinates are considered in the references just cited. For example, suppose that $K(\mathbf{x})=K\left(x_{2}\right)$ as in Sec. 3 here, and consider

$$
K\left(x_{2}\right) \equiv K\left(x_{2}, \alpha\right)=K_{0} \exp \left(-\alpha x_{2} / h\right),
$$

where $K_{0}>0$ is a constant having the same dimensions as $K$ and $\alpha \geq 0$ is a dimensionless constant. When $\alpha=0$ in (7.3), $K\left(x_{2}\right) \equiv K_{0}$ so that $\alpha$ provides a measure of the degree of inhomogeneity of the material. It is shown by Chan and Horgan [6] that the exact exponential decay rate for the steady-state problem for this example is

$$
\sqrt{k}=\sqrt{4 \pi^{2}+\alpha^{2}} /(2 h) .
$$


For $\alpha>0$, this decay rate is larger than that for the homogeneous material. The decay rate (7.4) is monotonically increasing in $\alpha$ so that the decay of end effects is faster as the material becomes more inhomogeneous. As discussed in the above references, the foregoing is true for the class of materials for which $\left(K^{1 / 2}\right)^{\prime \prime} \geq 0$ and

$$
\left[K^{1 / 2}\left(x_{2}, \alpha\right)\right]^{\prime \prime} K^{-1 / 2}\left(x_{2}, \alpha\right)
$$

is monotonic increasing in $\alpha$, where the prime denotes differentiation with respect to $x_{2}$. For $K(\mathbf{x})=K\left(x_{1}, x_{2}\right)$ as in Sec. 4, examples using several illustrative models for $K\left(x_{1}, x_{2}\right)$ that arise in the literature on functionally-graded materials are also provided by Chan and Horgan [6].

The sharper estimate (6.10) for the transient problem again involves the quantity $k$ so that the results of Chan and Horgan [6] may be directly used in (6.10). As pointed out at the end of Sec. 6, our main emphasis is on the decay factor (6.11) in which the only parameter appearing is the constant $d$ given by (6.12). As can be seen from (6.12), the constant $d$ depends on the material inhomogeneity in a very simple way. For the example (7.3), if we further assume that

$$
c \rho=c_{0} \rho_{0} \exp \left(-\beta x_{2} / h\right),
$$

where $\beta \geq 0$ is a dimensionless constant, then

$$
\kappa=\kappa\left(x_{2}\right)=\kappa_{0} \exp \left[-(\alpha-\beta) x_{2} / h\right]
$$

where

$$
\kappa_{0}=\frac{K_{0}}{\rho_{0} c_{0}}
$$

is a thermal diffusivity constant. Thus the decay factor (6.11) for the case $\alpha \geq \beta$ is simply

$$
\exp \left(-\frac{z^{2}}{4 \kappa_{0} t}\right)
$$

while for $\alpha<\beta$, the decay factor is

$$
\exp \left(-\frac{e^{(\alpha-\beta)} z^{2}}{4 \kappa_{0} t}\right)
$$

which yields a decay rate smaller than that in (7.8). These results reflect the interplay between the degree of inhomogeneity parameters $\alpha$ and $\beta$, i.e., the measures of inhomogeneity of $K\left(x_{2}\right)$ and $c\left(x_{2}\right) \rho\left(x_{2}\right)$ respectively. Other illustrative examples of the foregoing type may easily be constructed, where the exponentials in (7.3), (7.5) are replaced by powers of $x_{2}$ (cf. Carslaw and Jaeger [5], pp. 413-414). Thus, suppose that

$$
\begin{gathered}
K\left(x_{2}\right)=K_{0}\left(1+\alpha x_{2} / h\right)^{n}, \\
c\left(x_{2}\right) \rho\left(x_{2}\right)=c_{0} \rho_{0}\left(1+\alpha x_{2} / h\right)^{m},
\end{gathered}
$$

where $\alpha, n, m$ are nonnegative constants. The homogeneous case can be recovered on setting $\alpha=0$ or $n=m=0$. Then

$$
\kappa\left(x_{2}\right)=\kappa_{0}\left(1+\alpha x_{2} / h\right)^{n-m},
$$


where the constant thermal diffusivity is still given by (7.7). From (7.12), it follows that

$$
\begin{gathered}
\kappa_{M}=\kappa_{0}(1+\alpha)^{n-m}, \quad \text { if } n>m, \\
\kappa_{M}=\kappa_{0}, \quad \text { if } n \leq m
\end{gathered}
$$

Thus the decay factor (6.11) for the case where $n \leq m$ is again given by (7.8) while for $n>m$, it is

$$
\exp \left(-\frac{z^{2}(1+\alpha)^{m-n}}{4 \kappa_{0} t}\right),
$$

which again yields a decay rate which is smaller than that in (7.8).

Acknowledgments. The research of C.O.H. was supported by the U.S. National Science Foundation under Grant DMS-96-22748 and by the U.S. Air Force Office of Scientific Research under Grant AFOSR-F49620-98-1-0443. The work was initiated during a visit of C.O.H. to U.P.C., Terrassa, Barcelona, Spain in March 1999. The hospitality of the Department de Matematica Aplicada 2 at U.P.C. is gratefully acknowledged. The research of R.Q. was supported by the project BFM2000-0809 of the Ministry of Science and Technology of the Spanish Government.

\section{REFERENCES}

[1] M. S. Abid Mian and A. J. M. Spencer, Exact solutions for functionally graded laminated elastic materials, J. Mech. Phys. Solids 46, 2283-2295 (1998)

[2] M. Abramowitz and I. A. Stegun (eds.), Handbook of Mathematical Functions, Dover Publications, New York, 1965

[3] B. A. Boley, Upper bounds and Saint-Venant's principle in transient heat conduction, Quart. Appl. Math. 18, 205-207 (1960)

[4] B. A. Boley and J. H. Weiner, Theory of Thermal Stresses, John Wiley, New York, 1960

[5] H. S. Carslaw and J. C. Jaeger, Conduction of Heat in Solids, (2nd ed.), Oxford University Press, Oxford, 1959

[6] A. M. Chan and C. O. Horgan, End effects in anti-plane shear for an inhomogeneous isotropic linearly elastic semi-infinite strip, J. Elasticity 51, 227-242 (1998)

[7] F. Erdogan, Fracture mechanics of functionally graded materials, Composites Engineering 5, 753770 (1995)

[8] F. Franchi and B. Straughan, Spatial decay estimates and continuous dependence on modeling for an equation from dynamo theory, Proc. Roy. Soc. London A 445, 437-451 (1994)

[9] C. O. Horgan, Recent developments concerning Saint-Venant's principle: an update, Applied Mechanics Reviews 42, 295-303 (1989)

[10] C. O. Horgan, Recent developments concerning Saint-Venant's principle: a second update, Applied Mechanics Reviews 49, 101-111 (1996)

[11] C. O. Horgan and A. M. Chan, Torsion of functionally graded isotropic linearly elastic bars, J. of Elasticity 52, 181-199 (1999)

[12] C. O. Horgan and A. M. Chan, Vibration of inhomogeneous strings, rods and membranes, J. Sound and Vibration 225, 503-513 (1999)

[13] C. O. Horgan and J. K. Knowles, Recent developments concerning Saint-Venant's principle, In : J. W. Hutchinson (ed.), Advances in Applied Mechanics, Vol. 23, Academic Press, New York, 1983, pp. $179-269$

[14] C. O. Horgan and K. L. Miller, Anti-plane shear deformations for homogeneous and inhomogeneous anisotropic linearly elastic solids, J. Applied Mechanics 61, 23-29 (1994)

[15] C. O. Horgan and L. E. Payne, On the asymptotic behavior of solutions of linear second-order boundary value problems on a semi-infinite strip, Arch. Rational Mech. Anal. 124, 277-303 (1993) 
[16] C. O. Horgan, L. E. Payne, and L. T. Wheeler, Spatial decay estimates in transient heat conduction, Quart. Appl. Math. 42, 119-127 (1984)

[17] C. O. Horgan and R. Quintanilla, Saint-Venant end effects in anti-plane shear for functionally graded linearly elastic materials, Math. and Mechanics of Solids 6, 115-132 (2001)

[18] C. O. Horgan and L. T. Wheeler, Spatial decay estimates for the heat equation via the maximum principle, J. of Appl. Math. and Phys. (ZAMP) 27, 371-376 (1976)

[19] J. Ignaczak, Saint-Venant type decay estimates for transient heat conduction in a composite rigid semispace, J. of Thermal Stresses 21, 185-204 (1998)

[20] Z. H. Jin and R. C. Batra, Some basic fracture mechanics concepts in functionally graded materials, J. Mech. Phys. Solids 44, 1221-1235 (1996)

[21] J. K. Knowles, On the spatial decay of solutions of the heat equation, J. of Appl. Math. and Phys. (ZAMP) 227, 1050-1056 (1971)

[22] C. T. Loy, K. Y. Lam, and J. N. Reddy, Vibration of functionally graded cylindrical shells, Internat. J. Mech. Sciences 41, 309-324 (1999)

[23] J. Aboudi, M. J. Pindera, and S. M. Arnold, Higher-order theory for functionally graded materials, Composites: Part B, 30, 777-832 (1999)

[24] R. Quintanilla, Spatial behavior for nonlinear heat equations, Math. Models Meth. Appl. Sci. 7, 633-647 (1997)

[25] F. T. Rooney and M. Ferrari, Torsion and flexure of inhomogeneous elements, Composites Engineering 5, 901-911 (1995)

[26] M. R. Scalpato and C. O. Horgan, Saint-Venant decay rates for an isotropic inhomogeneous linearly elastic solid in anti-plane shear, Journal of Elasticity 48, 145-166 (1997) 